\title{
Convergence of a Penalty-Finite Element Approximation for an Obstacle Problem
}

\author{
Noboru Kikuchi \\ The University of Michigan, Ann Arbor, Michigan 48109, USA
}

\begin{abstract}
Summary. This study establishes an error estimate for a penalty-finite element approximation of the variational inequality obtained by a class of obstacle problems. By special identification of the penalty term, we first show that the penalty solution converges to the solution of a mixed formulation of the variational inequality. The rate of convergence of the penalization is $\varepsilon$ where $\varepsilon$ is the penalty parameter. To obtain the error of finite element approximation, we apply the results obtained by Brezzi, Hager and Raviart for the mixed finite element method to the variational inequality.
\end{abstract}

Subject Classifications: AMS(MOS): 65N30; CR: 5.17.

\section{Introduction}

This study establishes an error estimate for a penalty-finite element approximation of the variational inequality obtained by a class of obstacle problems for a membrane. We shall show convergence of the approximation with respect to the penalty parameter $\varepsilon$ and the mesh size $h$ of the finite element model.

Many works have been published on finite element approximations of variational inequalities together with a priori error estimates, e.g., Mosco and Strang [14], Falk [9, 10], Brezzi, Hager and Raviart [6, 7], and others. All these works are concerned with the primal formulation of variational inequalities, except that of Brezzi, Hager and Raviart [7] which provides general ideas and a methodology to obtain a priori error estimates for the mixed (Lagrangian multiplier) formulation of variational inequalities. For the penalty method, however, which is now widely used in the practical fields of engineering, there are few works. Bercovier [3] has obtained a priori estimates with respect to $\varepsilon$ and $h$ together, employing the study of the perturbed Lagrangian method (see Temam [16]). The work by Bercovier [3] deals with problems having constraints represented by equations such as incompressibility div $\mathbf{u}=0$ of Stokes' problems or linearly incompressible elasticity. 
In the present analysis, we will extend Bercovier's theory of obtaining a priori error estimates with respect to $\varepsilon$ and $h$ to a much wider class of problems represented by variational inequalities. As a model problem, a class of obstacle problems will be worked by the penalty-finite element approximation. A similar analysis would be applicable to other types of problems involving both equality and inequality constraints (see Oden, Kikuchi and Song [15]). In the case of equality constraints, the procedure to obtain the estimate for the penalty parameter $\varepsilon$ is suggested by Aubin [1].

It should be noted that direct methods, using projection maps from the whole space into constrained sets, work well for the obstacle problem discussed in this paper. However, physically important quantities such as contact pressure can be easily obtained by penalty methods, whereas direct methods cannot provide such quantities in general. Another advantage of penalty methods is their handy adaptation into existing computer codes for equations. Indeed, just a subroutine for the penalty terms must be added into the existing computer codes without any modifications in other parts, in order to solve variational inequalities.

We will first briefly review the penalty arguments for the obstacle problem, details of which can be found in Lions [12].

The next section will be devoted to the approximation of the variational inequality representing the obstacle problem by a penalty-finite element method together with numerical integration for the penalty term. We will show that the penalized solution $u_{\varepsilon}^{h}$ and the pressure $p_{\varepsilon}^{h}$, constructed by a special identification of the numerical integration of the penalty term, converge to the solutions $u^{h}$ and $p^{h}$ of a mixed (Lagrangian multiplier) formulation of the variational inequality. The convergence rate of the penalization will be obtained by assuming the discrete LBB (Ladyzhenskaya, Babuška, Brezzi) condition. The final estimates will be established by combining the analysis of mixed methods for variational inequalities obtained by Brezzi, Hager and Raviart [7].

\section{Obstacle Problems}

Let an elastic membrane $\bar{\Omega}$ be spanned over an obstacle. Let the height of the obstacle be given by $g(\mathbf{x}), \mathbf{x} \in \Omega$ and let the deflection of the membrane be given by $u$. Then the problem can be defined by the variational inequality

where

$$
u \in K: a(u, v-u) \geqq f(v-u), \quad \forall v \in K
$$

$$
\begin{gathered}
K=\left\{v \in H_{0}^{1}(\Omega): v-g \leqq 0 \text { a.e. in } \Omega\right\} \\
a(u, v)=\int_{\Omega} \mu(\mathbf{x}) \nabla u \cdot \nabla v d \mathbf{x} \\
f(v)=\int_{\Omega} f v d \mathbf{x} .
\end{gathered}
$$

Suppose that

$$
g \in H^{1}(\Omega), \quad f \in L^{\infty}(\Omega), \quad 0<\mu_{0} \leqq \mu(\mathbf{x}) \leqq \mu_{1} \quad \text { all } \mathbf{x} \in \Omega .
$$


Then, there exists a unique solution $u \in K$ to the variational inequality (2.1), and $u$ satisfies

provided that

$$
u \in H^{2}(\Omega)
$$

$$
g \in H^{2}(\Omega), \quad g \geqq 0 \quad \text { on } \Gamma .
$$

These results are found in [13] or [14].

The variational inequality $(2.1)$ is also resolved by penalty methods, and a characterization of the solution to (2.1) is obtained in terms of the "mixed" variational formulation. A general theory of the penalty method can be found in [12] or in a survey article [11].

Let $P_{K}$ be the projection of $H_{0}^{1}(\Omega)$ onto $K$, and $J$ be the duality map of $H_{0}^{1}(\Omega)$ into $H^{-1}(\Omega)$. A penalty operator $\beta$ of $H_{0}^{1}(\Omega)$ for the constrained set $K$ is defined by

so that

$$
\beta(u)=J\left(I-P_{K}\right)(u)
$$

$$
\langle\beta(u), v\rangle=0, \quad \forall v \in H_{0}^{1}(\Omega) \quad \text { if and only if } u \in K
$$

In the present problem, we may set

$$
\begin{aligned}
& \beta(u)=J\left((u-g)^{+}\right) \\
& \quad J=-\Delta+I \\
& \phi^{+} \in H_{0}^{1}(\Omega) \text { is defined by } \\
& \phi(x)^{+}=\operatorname{Max}(0, \phi(x)) \text { a.e. in } \Omega .
\end{aligned}
$$

The penalization of the variational inequality $(2.1)$ is then represented by

$$
u_{\varepsilon} \in H_{0}^{1}(\Omega): a\left(u_{\varepsilon}, v\right)+\frac{1}{\varepsilon}\left\langle\beta\left(u_{\varepsilon}\right), v\right\rangle=f(v), \quad \forall v \in H_{0}^{1}(\Omega)
$$

where $\langle\cdot, \cdot\rangle$ is the duality pairing on $H^{-1}(\Omega) \times H_{0}^{1}(\Omega)$. Applying a general convergence theorem for penalization (see [12; Theorem 5.2 in Chapter 3]), we can conclude the following results.

Theorem 2.1. Suppose that condition (2.5) holds. Then the sequence $\left\{u_{\varepsilon}\right\}$ of solutions of the penalization (2.11) converges weakly to the solution $u \in K$ of the variational inequality (2.1). Moreover, we have the estimate

$$
\left\|\beta\left(u_{\varepsilon}\right)\right\|_{1}^{*}=\left\|J\left(u_{\varepsilon}-g\right)^{+}\right\|_{1}^{*} \leqq C \varepsilon
$$

where \|\|$_{s}^{*}$ is the dual norm to the Sobolev norm $\|\cdot\|_{s}$ for $H^{s}(\Omega)$, and $C$ is a positive constant.

We now define the approximation of "contact pressure" by

$$
p_{\varepsilon}=-\frac{1}{\varepsilon} \beta\left(u_{\varepsilon}\right)
$$

We will show that $p_{\varepsilon}$ converges to the Lagrangian multiplier for the constraint $u-g \leqq 0$. Let

$$
N=\left\{q \in H^{-1}(\Omega): q \leqq 0\right\} .
$$


Theorem 2.2. Suppose that $g \in H_{0}^{1}(\Omega)$. Then the weak limit $u$ of solutions $u_{\varepsilon}$ of the penalization (2.11) and the weak limit $p$ of approximations $p_{\varepsilon}$ of the "contact pressure" satisfy the following system of inequalities:

$$
\begin{array}{cc}
u \in K: a(u, v)-\langle p, v\rangle=f(v), & \forall v \in H_{0}^{1}(\Omega) \\
p \in N:\langle q-p, u-g\rangle \geqq 0, & \forall q \in N .
\end{array}
$$

Proof. We know that the sequences $u_{\varepsilon}$ and $p_{\varepsilon}$ are uniformly bounded in $\varepsilon$. Since $a(\cdot, \cdot)$ is a continuous bilinear form, and since $\langle\cdot, \cdot\rangle$ is the duality pairing.

$$
a\left(u_{\varepsilon}, v\right)-\left\langle p_{\varepsilon}, v\right\rangle=f(v), \quad \forall v \in H_{0}^{1}(\Omega)
$$

converges to the equation

$$
a(u, v)-\langle p, v\rangle=f(v), \quad \forall v \in H_{0}^{1}(\Omega)
$$

where $p$ is the weak limit of $p_{\varepsilon}$, as $\varepsilon \rightarrow 0$. Suppose that $q \in N$. Then

$$
\left\langle q-p_{\varepsilon}, u_{\varepsilon}-g\right\rangle=\left\langle q, u_{\varepsilon}-g\right\rangle+\frac{1}{\varepsilon}\left\langle J\left(u_{\varepsilon}-g\right)^{+},\left(u_{\varepsilon}-g\right)^{+}\right\rangle \geqq\left\langle q, u_{\varepsilon}-g\right\rangle
$$

i.e.,

$$
\lim _{\varepsilon \rightarrow 0}\left\langle q-p_{\varepsilon}, u_{\varepsilon}-g\right\rangle \geqq\langle q, u-g\rangle \geqq 0
$$

On the other hand,

$$
\begin{gathered}
\left\langle q-p_{\varepsilon}, u_{\varepsilon}-g\right\rangle=\left\langle q, u_{\varepsilon}-g\right\rangle-a\left(u_{\varepsilon}, u_{\varepsilon}-g\right)+f\left(u_{\varepsilon}-g\right) \\
\limsup _{\varepsilon \rightarrow 0}\left\langle q-p_{\varepsilon}, u_{\varepsilon}-g\right\rangle=\langle q, u-g\rangle-\liminf _{\varepsilon \rightarrow 0} a\left(u_{\varepsilon}, u_{\varepsilon}\right)+a(u, g)+f(u-g) .
\end{gathered}
$$

Since $v \rightarrow a(v, v)$ is weakly lower semicontinuous,

$$
\limsup _{\varepsilon \rightarrow 0}\left\langle q-p_{\varepsilon}, u_{\varepsilon}-g\right\rangle \leqq\langle q, u-g\rangle-a(u, u-g)+f(u-g)=\langle q-p, u-g\rangle .
$$

Therefore, for every $q \in N$,

$$
\langle q-p, u-g\rangle \geqq 0
$$

Thus, we have shown that the variational inequality (2.1) can be characterized by the mixed (Lagrangian multiplier) method for resolution of the constraint $u-g \leqq 0$ through arguments of the penalty method.

Remark 2.1. Since strong convergence of $u_{\varepsilon}$ to $u$ in Theorem 2.1 has been proved [18, Chap. 1, Sect. 3.2], the proof of Theorem 2.2 can be simplified and shortened. The structure of the proof for Theorem 2.2, however, is applicable to the case that strong convergence of " $u_{\varepsilon} \rightarrow u$ " may not be obtained.

\section{Approximation by Penalty-Finite Element Methods}

We now consider a penalty-finite element approximation of the variational inequality (2.1). 
Let $V_{h}$ be a finite element approximation of the space $V=H_{0}^{1}(\Omega)$ which is spanned by piecewise polynomials, and let the domain $\Omega$ be a polygon so that $\bar{\Omega}$ can be covered by finite elements exactly.

Instead of discretizing the variational inequality (2.1) with the constrained set (2.2), we approximate the form similar to (2.11). Suppose that the constrained set $K$ is identified with the set $\tilde{K}=H_{0}^{1}(\Omega) \cap \hat{K}$ where

$$
\hat{K}=\left\{v \in L^{2}(\Omega): v-g \leqq 0 \text { a.e. in } \Omega\right\}
$$

Then the penalty formulation to the variational inequality (2.1) becomes

$$
u \in H_{0}^{1}(\Omega): a\left(u_{\varepsilon}, v\right)+\frac{1}{\varepsilon}\left(\hat{\beta}\left(u_{\varepsilon}\right), v\right)=f(v), \quad \forall v \in H_{0}^{1}(\Omega)
$$

where

$$
\hat{\beta}\left(u_{\varepsilon}\right)=\left(u_{\varepsilon}-g\right)^{+},
$$

and $(\cdot, \cdot)$ is the $L^{2}$-inner product. We shall discretize (3.2) instead of (2.11) by finite element methods. In this case, while the operator $\hat{\beta}$ is "almost" linear, the term $\left(\hat{\beta}\left(u_{\varepsilon}^{h}\right), v^{h}\right)$ cannot be evaluated exactly for approximations $u_{\varepsilon}^{h}$ and $v^{h}$ in $V_{h}$, in general. In order to overcome this difficulty, we apply techniques of numerical integration. Then the following finite element approximation to (3.2) can be defined:

$$
u_{\varepsilon}^{h} \in V_{h}: a\left(u_{\varepsilon}^{h}, v^{h}\right)+\frac{1}{\varepsilon} I\left(\widehat{\beta}\left(u_{\varepsilon}^{h}\right), v^{h}\right)=f\left(v^{h}\right), \quad \forall v^{h} \in V_{h}
$$

where " $I$ " is the operation of numerical quadrature:

$$
I(g)=\sum_{e=1}^{E} \sum_{i=1}^{G} w_{i}^{e} g\left(\mathbf{x}_{i}^{e}\right)
$$

$e$ indicates the finite element, and $\mathbf{x}_{i}^{e}$ is the local coordinate of the $i$-th integration point within the $e$-th finite element. Suppose that

$$
w_{i}^{e} \geqq 0, \quad \forall i=1, \ldots, G
$$

i.e., the weights of numerical integration are all positive.

The formulation that the penalty term is constructed by the $L^{2}$-inner product in (3.4) instead of the duality pairing $\langle\cdot \cdot\rangle$ on $H^{-1}(\Omega) \times H_{0}^{1}(\Omega)$ is supported by the fact that for fixed $h$ the approximation of (2.1) is defined on a subset of a finite dimensional space $V_{h}$, which is naturally identified with its dual. The penalty for the constraint in approximation need be considered only on the space $V_{h}$ and convergence of $u_{\varepsilon}^{h}$ as $\varepsilon \rightarrow 0$ is discussed for fixed $h$. Then convergence of $u^{h}$ to $u$ occurs as $h \rightarrow 0$ in the infinite dimensional space $V=H_{0}^{1}(\Omega)$.

Because of the numerical integration of the penalty term in (3.4), we control the constraint $u^{h}-g \leqq 0$ pointwise, i.e., the penalization

$$
\frac{1}{\varepsilon} I\left(\hat{\beta}\left(u_{\varepsilon}^{h}\right), v^{h}\right)=\frac{1}{\varepsilon} \sum_{e=1}^{E} \sum_{i=1}^{G} w_{i}^{e}\left(u_{\varepsilon}^{h}-g\right)\left(\mathbf{x}_{i}^{e}\right)^{+} v^{h}\left(\mathbf{x}_{i}^{e}\right)
$$


implies that the penalty solution $u_{\varepsilon}^{h}$ almost satisfies the constraint $u_{\varepsilon}^{h}(\mathbf{x})-g \leqq 0$ at points of numerical integration, and that the quantity

$$
-\frac{1}{\varepsilon}\left(u_{\varepsilon}^{h}-g\right)\left(\mathbf{x}_{i}^{e}\right)^{+}
$$

corresponds to the approximation of "contact pressure" at $\mathbf{x}_{i}^{e}$ due to the obstacle. In order to convert pointwise information obtained by (3.4) to continuous information, we introduce the following rules of identification:

(i) let $p_{\varepsilon}^{h}$ be a piecewise polynomial spanned by Lagrangian interpolations associated with points of numerical integration, i.e., $p_{\varepsilon}^{h} \in Q_{h}$,

$$
p_{\varepsilon}^{h}\left(\mathbf{x}_{i}^{e}\right)=-\frac{1}{\varepsilon}\left(u_{\varepsilon}^{h}-g\right)\left(\mathbf{x}_{i}^{e}\right)^{+}, \quad 1 \leqq i \leqq G, 1 \leqq e \leqq E .
$$

The above two conditions imply the equation

$$
I\left(p_{\varepsilon}^{h}, v^{h}\right)=-I\left(\frac{1}{\varepsilon} \hat{\beta}\left(u_{\varepsilon}^{h}\right), v^{h}\right) .
$$

Then the penalty formulation (3.4) can be identified with

$$
u_{\varepsilon}^{h} \in V_{h}: a\left(u_{\varepsilon}^{h}, v^{h}\right)-I\left(p_{\varepsilon}^{h}, v^{h}\right)=f\left(v^{h}\right), \quad \forall v^{h} \in V_{h} .
$$

The piecewise polynomial $p_{\varepsilon}^{h}$ may be an approximation of the contact pressure.

Example 1. Let $\Omega \subset \mathbb{R}^{2}$, and let three-node linear triangular elements be used to construct the approximate space $V_{h}$. If $w_{i}^{e}=$ constant, and if $\mathbf{x}_{i}^{e}$ is the coordinate of the $i$-th nodal point of a triangular element, $p_{\varepsilon}^{h}$ is a piecewise linear polynomial as $v^{h} \in V_{h}$ is.

If 4-node quadrilateral isoparametric elements are used for $V_{h}$, and if the two-point trapezoid rule is applied to each direction $x_{1}$ and $x_{2}$ for the numerical integration, $p_{\varepsilon}^{h}$ becomes a piecewise bilinear polynomial as $v^{h} \in V_{h}$ is.

For 9-node quadrilateral isoparametric element, if the Simpson rule is applied to each direction $x_{1}$ and $x_{2}, p_{\varepsilon}^{h}$ is a piecewise bi-quadratic polynomial.

In the above choices, each $p_{\varepsilon}^{h}$ becomes conforming. Let

We briefly indicate how the nonlinear equation (3.4) is solved numerically.

$$
\hat{\beta}^{t}\left({ }^{t} u_{\varepsilon}^{h}\right)= \begin{cases}\widehat{\beta}\left({ }^{t} u_{\varepsilon}^{h}\right) & \text { if }\left({ }^{t-1} u_{\varepsilon}^{h}-g\right)\left(\mathbf{x}_{i}^{e}\right)>0 \\ 0 & \text { if }\left({ }^{t-1} u_{\varepsilon}^{h}-g\right)\left(\mathbf{x}_{i}^{e}\right) \leqq 0\end{cases}
$$

The penalty problem (3.4) can be solved by the successive iteration

$$
{ }^{t} u_{\varepsilon}^{h} \in V_{h}: a\left({ }^{t} u_{\varepsilon}^{h}, v^{h}\right)+\frac{1}{\varepsilon} I\left[\hat{\beta}^{t}\left({ }^{t} u_{\varepsilon}^{h}\right), v^{h}\right]=f\left(v^{h}\right), \quad \forall v^{h} \in V_{h} .
$$

It is expected that ${ }^{t} u_{\varepsilon}^{h} \rightarrow u_{\varepsilon}^{h}$ as $t \rightarrow+\infty$. 
Let $N_{h}$ be a closed convex subset of $Q_{h}$ defined by

$$
N_{h}=\left\{q^{h} \in Q_{h}: q^{h}\left(\mathbf{x}_{i}^{e}\right) \leqq 0,1 \leqq i \leqq G, 1 \leqq e \leqq E\right\}
$$

The set $N_{h}$ is the admissible set of contact pressure in the finite dimensional space. Using this we define the approximation of the constrained set (2.2): for $g \in C(\bar{\Omega})$,

$$
K_{h}=\left\{v^{h} \in V_{h}: I\left(q^{h}, v^{h}-g\right) \geqq 0, \forall q^{h} \in N_{h}\right\} .
$$

Theorem 3.1. Let $g \in C(\bar{\Omega})$. The solution $u_{\varepsilon}^{h}$ of the penalty form (3.4) is uniformly bounded in $\varepsilon$, and converges as $\varepsilon \rightarrow 0$ to a solution $u^{h}$ of the variational inequality

$$
u^{h} \in K_{h}: a\left(u^{h}, v^{h}-u^{h}\right) \geqq f\left(v^{h}-u^{h}\right), \quad \forall v^{h} \in K_{h} .
$$

Proof. (Uniform boundedness) Let $v^{h} \in K_{h}$. From (3.4),

$$
a\left(u_{\varepsilon}^{h}, v^{h}-u_{\varepsilon}^{h}\right)+\frac{1}{\varepsilon} I\left(\widehat{\beta}\left(u_{\varepsilon}^{h}\right), v^{h}-u_{\varepsilon}^{h}\right)=f\left(v^{h}-u_{\varepsilon}^{h}\right) .
$$

Since $\hat{\beta}\left(v^{h}\right)=0$,

$$
\begin{aligned}
I\left(\hat{\beta}\left(u_{\varepsilon}^{h}\right), v^{h}-u_{\varepsilon}^{h}\right) & =-I\left(\hat{\beta}\left(v^{h}\right)-\hat{\beta}\left(u_{\varepsilon}^{h}\right), v^{h}-u_{\varepsilon}^{h}\right) \\
& \leqq-I\left(\left(v^{h}-g\right)^{+}-\left(u_{\varepsilon}^{h}-g\right)^{+},\left(v^{h}-g\right)^{+}-\left(u_{\varepsilon}^{h}-g\right)^{+}\right) \leqq 0 .
\end{aligned}
$$

Thus,

$$
a\left(u_{\varepsilon}^{h}, v^{h}-u_{\varepsilon}^{h}\right) \geqq f\left(v^{h}-u_{\varepsilon}^{h}\right), \quad \forall v^{h} \in K_{h} .
$$

This further implies

i.e.,

$$
c \mu_{0}\left\|u_{\varepsilon}^{h}\right\|_{1} \leqq \mu_{1}\left\|u_{\varepsilon}^{h}\right\|_{1}\left\|v^{h}\right\|_{1}+\|f\|_{0}\left(\left\|v^{h}\right\|_{1}+\left\|u_{\varepsilon}^{h}\right\|_{1}\right)
$$

$$
\left\|u_{\varepsilon}^{h}\right\|_{1} \leqq C<+\infty
$$

(Convergence) Since $V_{h}$ is a finite dimensional space, there is a subsequence of $u_{\varepsilon}^{h}$, still denoted by $u_{\varepsilon}^{h}$, which converges to $u^{h}$ in $V^{h}$. Passing to the limit $\varepsilon \rightarrow 0$ in (3.15), we have

$$
a\left(u^{h}, v^{h}-u^{h}\right) \geqq f\left(v^{h}-u^{h}\right), \quad \forall v^{h} \in K_{h} .
$$

Thus, we need show only $u^{h} \in K_{h}$. Putting $v^{h}-u_{\varepsilon}^{h}$ instead of $v^{h}$ in (3.4),

$$
a\left(u_{\varepsilon}^{h}, v^{h}-u_{\varepsilon}^{h}\right)+\frac{1}{\varepsilon} I\left(\left(u_{\varepsilon}^{h}-g\right)^{+}, v^{h}-u_{\varepsilon}^{h}\right)=f\left(v^{h}-u_{\imath}^{h}\right) .
$$

If $v^{h} \in K_{h}$, i.e. if $\left(v^{h}-g\right)\left(\mathbf{x}_{i}^{e}\right) \leqq 0$,

Then

$$
\begin{aligned}
I\left(\left(u_{\varepsilon}^{h}-g\right)^{+}, v^{h}-u_{\varepsilon}^{h}\right) & =I\left(\left(u_{\varepsilon}^{h}-g\right)^{+}, v^{h}-g-\left(u_{\varepsilon}^{h}-g\right)\right) \\
& \leqq-I\left(\left(u_{\varepsilon}^{h}-g\right)^{+},\left(u_{\varepsilon}^{h}-g\right)^{+}\right) .
\end{aligned}
$$

$$
\begin{aligned}
I\left(\left(u_{\varepsilon}^{h}-g\right)^{+},\left(u_{\varepsilon}^{h}-g\right)^{+}\right) & \leqq \varepsilon\left\{a\left(u_{\varepsilon}^{h}, v^{h}-u_{\varepsilon}^{h}\right)-f\left(v^{h}-u_{\varepsilon}^{h}\right)\right\} \\
& \leqq \varepsilon\left\{a\left(u_{\varepsilon}^{h}, v^{h}\right)-f\left(v^{h}-u_{\varepsilon}^{h}\right)\right\} \\
& \leqq \varepsilon\left\{\mu_{1}\left\|u_{\varepsilon}^{h}\right\|_{1}\left\|v^{h}\right\|_{1}+\|f\|_{0}\left(\left\|v^{h}\right\|_{1}+\left\|u_{\varepsilon}^{h}\right\|_{1}\right)\right\}
\end{aligned}
$$


Since $u_{\varepsilon}^{h}$ is uniformly bounded, we can conclude that

$$
\left|\left(u_{\varepsilon}^{h}-g\right)\left(\mathbf{x}_{i}^{e}\right)^{+}\right|^{2} \leqq C \varepsilon \quad \text { and then } \quad\left(u^{h}-g\right)\left(\mathbf{x}_{i}^{e}\right) \leqq 0 .
$$

We will show that $p_{\varepsilon}^{h}$ also converges to $p^{h}$ in $Q_{h}$ as $\varepsilon \rightarrow 0$, as well as $u_{\varepsilon}^{h} \rightarrow u^{h}$, under an additional condition.

Theorem 3.2. Suppose that

$$
\exists \alpha_{h}>0: \alpha_{h}\left\|q^{h}\right\|_{0} \leqq \sup _{v^{h} \in V_{h}} \frac{I\left(q^{h}, v^{h}\right)}{\left\|v^{h}\right\|_{1}}, \quad \forall q^{h} \in Q_{h} .
$$

Then $p_{\varepsilon}^{h}$ is uniformly bounded in $\varepsilon$, and converges to $p^{h} \in Q_{h}$ which satisfies

$$
\begin{cases}a\left(u^{h}, v^{h}\right)-I\left(p^{h}, v^{h}\right)=f\left(v^{h}\right), & \forall v^{h} \in V_{h} \\ I\left(q^{h}-p^{h}, u^{h}-g\right) \geqq 0, & \forall q^{h} \in N_{h} .\end{cases}
$$

Proof. From (3.10) and (3.18),

$$
\alpha_{h}\left\|p_{\varepsilon}^{h}\right\|_{0} \leqq \sup _{v^{h} \in V_{h}} \frac{I\left(p_{\varepsilon}^{h}, v^{h}\right)}{\left\|v^{h}\right\|_{1}}=\sup _{v^{h} \in V_{h}} \frac{a\left(u_{\varepsilon}^{h}, v^{h}\right)-f\left(v^{h}\right)}{\left\|v^{h}\right\|_{1}} \leqq \mu_{1}\left\|u_{\varepsilon}^{h}\right\|_{1}+\|f\|_{0}
$$

i.e.,

$$
\left\|p_{\varepsilon}^{h}\right\|_{0} \leqq C / \alpha_{h}<+\infty
$$

Then there is a subsequence of $p_{\varepsilon}^{h}$, still denoted by $p_{\varepsilon}^{h}$, which converges to $p^{h}$. Passing to the limit $\varepsilon \rightarrow 0$ in (3.10) yields (3.19) $)_{1}$.

Applying (3.9), we have

$$
I\left(q^{h}-p_{\varepsilon}^{h}, u_{\varepsilon}^{h}-g\right)=I\left(q^{h}, u_{\varepsilon}^{h}-g\right)+\frac{1}{\varepsilon} I\left(\left(u_{\varepsilon}^{h}-g\right)^{+}, u_{\varepsilon}^{h}-g\right) \geqq I\left(q^{h}, u_{\varepsilon}^{h}-g\right) .
$$

Passing to the limit $\varepsilon \rightarrow 0$,

$$
I\left(q^{h}-p^{h}, u^{h}-g\right) \geqq I\left(q^{h}, u^{h}-g\right) \geqq 0, \quad \forall q^{h} \in N_{h}
$$

since $u^{h} \in K_{h}$.

We next find the rates of convergence $u_{\varepsilon}^{h} \rightarrow u^{h}$ and $p_{\varepsilon}^{h} \rightarrow p^{h}$ as $\varepsilon \rightarrow 0$.

Theorem 3.3. Suppose that conditions in Theorem 3.1 and 3.2 hold. Further suppose that

$$
I\left(p^{h}, q^{h}\right) \leqq C\left\|p^{h}\right\|_{0}\left\|q^{h}\right\|_{0}, \quad \forall p^{h}, q^{h} \in Q_{h} .
$$

Then there exists a positive constant $C$ such that

$$
\left\|u_{\varepsilon}^{h}-u^{h}\right\|_{1} \leqq C\left\|p^{h}\right\|_{0}\left(\varepsilon / \alpha_{h}\right), \quad\left\|p_{\varepsilon}^{h}-p^{h}\right\|_{0} \leqq C\left\|p^{h}\right\|_{0}\left(\varepsilon / \alpha_{h}^{2}\right) .
$$

Proof. From (3.10) and (3.19)

$$
a\left(u_{\varepsilon}^{h}-u^{h}, v^{h}\right)=I\left(p^{h}-p_{\varepsilon}^{h}, v^{h}\right), \quad \forall v^{h} \in V_{h} .
$$


Putting $v^{h}=u_{\varepsilon}^{h}-u^{h}$,

$$
a\left(u_{\varepsilon}^{h}-u^{h}, u_{\varepsilon}^{h}-u^{h}\right)=I\left(p_{\varepsilon}^{h}-p^{h}, u_{\varepsilon}^{h}-g\right)-I\left(p_{\varepsilon}^{h}-p^{h}, u^{h}-g\right) \leqq I\left(p_{\varepsilon}^{h}-p^{h}, u_{\varepsilon}^{h}-g\right)
$$

since $I\left(p^{h}, u^{h}-g\right)=0$ and $I\left(p_{\varepsilon}^{h}, u^{h}-g\right) \geqq 0$. Using the relation

$$
\begin{gathered}
\phi=\phi^{+}-\phi^{-} \quad \text { with } \phi^{-}=\operatorname{Max}\{0,-\phi\} \quad \text { for } \phi \in \mathbb{R}, \\
\begin{aligned}
I\left(p_{\varepsilon}^{h}-p^{h}, u_{\varepsilon}^{h}-g\right) & =I\left(p_{\varepsilon}^{h}-p^{h},\left(u_{\varepsilon}^{h}-g\right)^{+}\right)-I\left(p_{\varepsilon}^{h}-p^{h},\left(u_{\varepsilon}^{h}-g\right)^{-}\right) \\
& \leqq I\left(p_{\varepsilon}^{h}-p^{h},-\varepsilon p_{\varepsilon}^{h}\right) \leqq C \varepsilon\left\|p_{\varepsilon}^{h}-p^{h}\right\|_{0}\left\|p^{h}\right\|_{0} .
\end{aligned}
\end{gathered}
$$

Thus, we have

$$
c \mu_{0}\left\|u_{\varepsilon}^{h}-u^{h}\right\|_{1}^{2} \leqq a\left(u_{\varepsilon}^{h}-u^{h}, u_{\varepsilon}^{h}-u^{h}\right) \leqq C\left\|p_{\varepsilon}^{h}-p^{h}\right\|_{0}\left\|p^{h}\right\|_{0} \varepsilon .
$$

From (3.23) and the assumption (3.18)

$$
\alpha_{h}\left\|p_{\varepsilon}^{h}-p^{h}\right\|_{0} \leqq \sup \frac{I\left(p_{\varepsilon}^{h}-p^{h}, v^{h}\right)}{\left\|v^{h}\right\|_{1}} \leqq \mu_{1}\left\|u_{\varepsilon}^{h}-u^{h}\right\|_{1} .
$$

Combining (3.24) and (3.25) yields the estimates (3.22).

The condition (3.18) is called the discrete $L B B$ condition after Ladyzhenskaya, Babuška [2] and Brezzi [5]. Under this assumption, we have shown that the discrete penalization (3.4) converges to the discrete "mixed" (or Lagrangian multiplier) formulation $(3.19)$ with rate of convergence $\varepsilon$ with respect to the penalty parameter.

Example 2. The condition (3.18) will be proved for triangular 3-node linear finite elements. Let

$$
I\left(q^{h}, v^{h}\right)=\sum_{e=1}^{E} \sum_{i=1}^{3} w_{i}^{e} q^{h}\left(\mathbf{x}_{i}^{e}\right) v^{h}\left(\mathbf{x}_{i}^{e}\right)
$$

where $w_{i}^{e}=\operatorname{mes}\left(\Omega_{e}\right) / 3, \operatorname{mes}\left(\Omega_{e}\right)$ is the area of the $e$-th finite element, $x_{i}^{e}$ coincides to the coordinates of nodal points of the element, and $i=1,2$, and 3 . That is, the following rules are supposed within the master element for 3-node triangular elements, (Fig. 1).

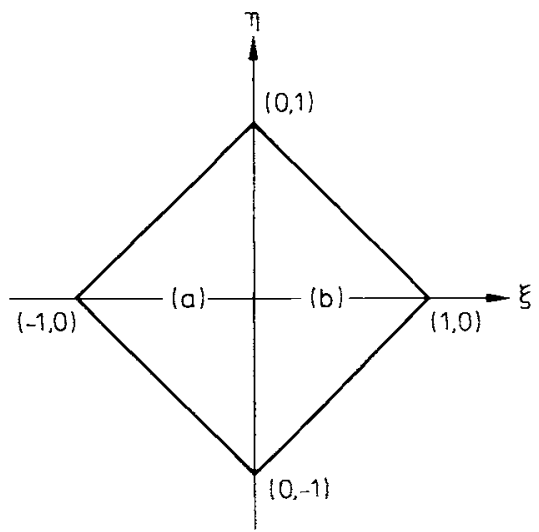

Fig. 1. Master elements of linear triangular finite elements 


$$
\begin{array}{lll}
\mathbf{x}_{1}^{e}=(-1,0) & & \mathbf{x}_{1}^{e}=(1,0) \\
\mathbf{x}_{2}^{e}=(0,-1) & \text { for }(a) & \mathbf{x}_{e}^{2}=(0,-1) \quad \text { for }(b) \\
\mathbf{x}_{3}^{e}=(0,1) & & \mathbf{x}_{3}^{e}=(0,1) .
\end{array}
$$

Then, we have following relations in an element related to the $(a)$-master element:

$$
\begin{aligned}
q^{h}(\mathbf{x}) & =q_{0}+q_{i} \xi+q_{2} \eta \\
I\left(q^{h}, q^{h}\right) & =\frac{1}{3}\left[\left(q_{0}-q_{1}\right)^{2}+2 q_{0}^{2}+2 q_{1}^{2}\right] \operatorname{mes}\left(\Omega_{e}\right) \\
\left\|q^{h}\right\|_{0, \Omega_{e}}^{2} & =\left(q^{h}, q^{h}\right)=\frac{1}{3}\left[\left(q_{0}-q_{1}\right)^{2}+2 q_{0}^{2}-\frac{1}{2} q_{1}^{2}+\frac{1}{2} q_{2}^{2}\right] \operatorname{mes}\left(\Omega_{e}\right) .
\end{aligned}
$$

Similar expansions for $\Omega_{e}$ related to the $(b)$-master element yield similar results to $(3.27)$. Then we have

$$
I\left(q^{h}, q^{h}\right) \geqq\left\|q^{h}\right\|_{0}^{2} \quad \text { and } \quad I\left(v^{h}, v^{h}\right) \geqq\left\|v^{h}\right\|_{0}^{2}
$$

since $v^{h} \in V_{h}$ is also linear within an element.

Taking the special relation

in (3.26) yields

$$
q^{h}\left(\mathbf{x}_{i}^{e}\right)=v_{q}^{h}\left(\mathbf{x}_{i}^{e}\right), \quad i=1,2,3 ; e=1, \ldots, E
$$

$$
I\left(q^{h}, v_{q}^{h}\right)=\left(I\left(q^{h}, q^{h}\right)\right)^{\frac{1}{2}}\left(I\left(v_{q}^{h}, v_{q}^{h}\right)\right)^{\frac{1}{2}} .
$$

Applying (3.28) in (3.29) implies

$$
I\left(q^{h}, v_{q}^{h}\right) \geqq\left\|q^{h}\right\|_{0}\left\|v_{q}^{h}\right\|_{0} .
$$

Assuming the inverse property of $V_{h}$ :

$$
\left\|v_{h}\right\|_{1} \leqq C_{i} h^{-1}\left\|v^{h}\right\|_{0}, \quad \forall v^{h} \in V_{h}
$$

it can be concluded that

and then

$$
I\left(q^{h}, v_{q}^{h}\right) \geqq \frac{1}{C_{i}} h\left\|v_{q}^{h}\right\|,\left\|q^{h}\right\|_{0}
$$

$$
\sup _{v^{h} \in V_{h}} \frac{I\left(q^{h}, v^{h}\right)}{\left\|v^{h}\right\|_{1}} \geqq \frac{1}{C_{i}} h\left\|q^{h}\right\|_{0} .
$$

Therefore, $\alpha_{h}$ in (3.18) is $h / C_{i}$ for the above choice.

Example 3. For the 4-node quadrilateral isoparametric element and the trapezoid rule for numerical integration, the condition (3.18) can be obtained by arguments similar to Example 2. If $\Omega_{e}$ is assumed to be the master element, we have

$$
\begin{aligned}
q^{h}(\mathbf{x}) & =q_{0}+q_{1} \xi+q_{2} \eta+q_{3} \xi \eta \\
I\left(q^{h}, q^{h}\right) & =\left(q_{0}^{2}+q_{1}^{2}+q_{2}^{2}+q_{3}^{2}\right) \operatorname{mes}\left(\Omega_{e}\right) \\
\left\|q^{h}\right\|_{0 \Omega_{e}}^{2} & =\left(q_{0}^{2}+\frac{1}{3} q_{l}^{2}+\frac{1}{3} q_{2}^{2}+\frac{1}{9} q_{3}^{2}\right) \operatorname{mes}\left(\Omega_{e}\right) .
\end{aligned}
$$


Then

$$
I\left(q^{h}, q^{h}\right) \geqq\left\|q^{h}\right\|_{0}^{2} \quad \text { and } \quad I\left(v^{h}, v^{h}\right) \geqq\left\|v^{h}\right\|_{0}^{2} .
$$

Thus, using the inverse property (3.30), we have

$$
\sup _{v^{h} \in Y_{h}} \frac{\left(q^{h}, v^{h}\right)}{\left\|v^{h}\right\|} \geqq \frac{1}{C_{i}} h\left\|q^{h}\right\|_{0} .
$$

If the one-point Gaussian rule for numerical integration with piecewise constant contact pressure $q^{h}$ is applied, the condition (3.18) may not hold. In fact, the choice

$$
q^{h}\left(\mathbf{x}_{i}^{e}\right)=v_{q}^{h}\left(\mathbf{x}_{i}^{e}\right) \quad \text { yields } \quad I\left(q^{h}, v_{q}^{h}\right)=\sqrt{I\left(q^{h}, q^{h}\right)} \sqrt{I\left(v_{q}^{h}, v_{q}^{h}\right)} .
$$

Since $q^{h}$ is constant within an element,

However,

$$
I\left(q^{h}, q^{h}\right)=\left\|q^{h}\right\|_{0}^{2} .
$$

and

$$
I\left(v_{q}^{h}, v_{q}^{h}\right)=\left(v_{q}^{h}\right)_{0}^{2} \operatorname{mes} \Omega_{e}
$$

$$
\left\|v_{q}^{h}\right\|_{0, \Omega_{e}}^{2}=\left(\left(v_{q}^{h}\right)_{0}^{2}+\frac{1}{3}\left(v_{q}^{h}\right)_{1}^{2}+\frac{1}{3}\left(v_{q}^{h}\right)_{2}^{2}+\frac{1}{9}\left(v_{q}^{h}\right)_{3}^{2}\right) \operatorname{mes} \Omega_{e}
$$

within an element. This means that, for some $C_{e}>0$,

$$
-C_{e} h^{2}\left\|v_{q}^{h}\right\|_{\mathrm{t}}^{2}+\left\|v_{q}^{h}\right\|_{0}^{2} \leqq C I\left(v_{q}^{h}, v_{q}^{h}\right) .
$$

Thus, (3.18) need not be true, in general.

Now, it is necessary for full estimates of $u_{t}^{h}$ and $u$ to find $h$-estimates between the limits $\left(u^{h}, p^{h}\right)$ and the solutions $(u, p)$ of the "mixed" formulation (2.15). To this end, we have to recall the study by Brezzi, Hager and Raviart [7] for finite element analysis of the mixed type of variational inequalities.

\section{Estimates for the Mixed Method}

Let $(u, p) \in K \times N$ be the solution of the mixed formulation (2.15). Let $\left(u^{h}, p^{h}\right) \in K_{h}$ $\times N_{h}$ be the limit of $\left\{u_{\varepsilon}^{h}, p_{\varepsilon}^{h}\right\}$ as $\varepsilon \rightarrow 0$ which satisfies the discrete mixed formulation (3.19).

Let $E_{l}$ be the error of numerical integration $I$ defined by (3.5) such that

for every $f, g \in C(\bar{\Omega})$.

$$
E_{l}(f, g)=(f, g)-I(f, g)
$$

Theorem 4.1. Suppose that the following regularity holds:

$$
u \in H^{s}(\Omega), \quad g \in H^{s}(\Omega), \quad p \in H^{s-2}(\Omega), \quad s \geqq 2 .
$$

Then the estimate

$$
\begin{aligned}
\left\|u-u^{h}\right\|_{1}^{2} \leqq & C_{1}\left\{\left\|u-v^{h}\right\|_{1}^{2}+\left(\left\|p-q^{h}\right\|_{1}^{*}\right)^{2}+\left\|p-q^{h}\right\|_{s}^{*}\right. \\
& \left.+\left\|u-v^{h}\right\|_{s-2}^{*}+\mid E_{I}\left(q^{h}, u^{h}-g\right) \|\right\}
\end{aligned}
$$


holds for every $v^{h} \in K_{h}$ and $q^{h} \in N_{h}$, where $C_{1}$ depends only upon $\|u\|_{s}$ and $\|p\|_{s-2}$. Moreover, the estimate

$$
\begin{aligned}
\left\|u-u^{h}\right\|_{1}^{2} \leqq & C_{2}\left\{\left\|u-v^{h}\right\|_{1}^{2}+\left(\left\|p-q^{h}\right\|_{1}^{*}\right)^{2}+\left\|p-p^{h}\right\|_{0}\left\|u-v^{h}\right\|_{0}\right. \\
& \left.+\left\|p-q^{h}\right\|_{s}^{*}+\left|E_{I}\left(q^{h}, u^{h}-s\right)\right|+\left|E_{I}\left(p^{h}, v^{h}-u\right)\right|\right\}
\end{aligned}
$$

holds for every $v^{h} \in V_{h}$ and $q^{h} \in N_{h}$, where $C_{2}$ is independent of the mesh size $h$ of the finite element model.

Proof. From (2.15) and (3.19),

$$
a\left(u-u^{h}, v^{h}\right)=\left(p, v^{h}\right)-I\left(p^{h}, v^{h}\right), \quad \forall v^{h} \in V_{h} .
$$

Applying (4.5), we have

$$
\begin{aligned}
a\left(u-u^{h}, u-u^{h}\right)= & a\left(u-u^{h}, u-v^{h}\right)+\left(p, v^{h}-u^{h}\right)-I\left(p^{h}, v^{h}-u^{h}\right) \\
\leqq & a\left(u-u^{h}, u-v^{h}\right)+\left(p-q^{h}, u-u^{h}\right)+\left(q^{h}-p, u-g\right) \\
& +\left(p, v^{h}-u\right)-E_{l}\left(q^{h}, u^{h}-g\right)-I\left(p^{h}, v^{h}-g\right)-I\left(q^{h}, u^{h}-g\right)
\end{aligned}
$$

for every $v^{h} \in V_{h}$ and $q^{h} \in N_{h}$. If $v^{h} \in K_{h}$ and $q^{h} \in N_{h}$, then

$$
\begin{aligned}
a\left(u-u^{h}, u-u^{h}\right) \leqq & a\left(u-u^{h}, u-v^{h}\right)+\left(p-q^{h}, u-u^{h}\right) \\
& +\left(q^{h}-p, u-g\right)+\left(p, v^{h}-u\right)-E_{I}\left(q^{h}, u^{h}-g\right) .
\end{aligned}
$$

Since $a(\cdot, \cdot)$ is coercive and continuous, regularity assumption (4.2) and Young's inequality imply the estimate (4.3).

Furthermore, from (4.6), we can obtain the inequality

$$
\begin{aligned}
a\left(u-u^{h}, u-u^{h}\right)= & a\left(u-u^{h}, u-v^{h}\right)+\left(p-q^{h}, u-u^{h}\right)+\left(p-p^{h}, v^{h}-u\right) \\
& +\left(q^{h}-p, u-g\right)-E_{I}\left(q^{h}, u^{h}-g\right)+E_{I}\left(p^{h}, v^{h}-u\right) \\
& -I\left(q^{h}, u^{h}-g\right)-I\left(p^{h}, u-g\right) \\
\leq & a\left(u-u^{h}, u-v^{h}\right)+\left(p-q^{h}, u-u^{h}\right)+\left(p-p^{h}, v^{h}-u\right) \\
& +\left(q^{h}-p, u-g\right)-E_{I}\left(q^{h}, u^{h}-g\right)+E_{I}\left(p^{h}, v^{h}-u\right)
\end{aligned}
$$

for every $q^{h} \in N_{h}$ and $v^{h} \in V_{h}$. Then the estimate (4.4) follows from coerciveness and continuity of $a(\cdot \cdot \cdot),(4.2)$, and Young's inequality.

It is noted that the estimate (4.3) is constructed in subsets $K_{h}$ and $N_{h}$ of $V_{h}$ and $Q_{h}$, respectively. Then, interpolation errors of finite element methods must be discussed in restricted sets $K_{h}$ and $N_{h}$. The estimate (4.4) is implied within $V_{h}$ and $N_{h}$. However, the term $\left\|p-p_{h}\right\|_{0}$ is included in the right-hand side of (4.4), and is estimated as follows:

Theorem 4.2. Suppose that there exists a positive number $\hat{\alpha}_{h}$ such that

$$
\hat{x}_{h}\left\|q^{h}\right\|_{0} \leqq \sup _{v^{h} \in V_{h}} \frac{\left(q^{h}, v^{h}\right)}{\left\|v^{h}\right\|_{1}}, \quad v^{h} \neq 0, q^{h} \in Q_{h} .
$$


Then the estimate

$$
\left\|p-p^{h}\right\|_{0}^{2} \leqq C_{2}\left\{\left(\left\|u-u^{h}\right\|_{1} / \hat{\alpha}_{h}\right)^{2}+\left\|p-q^{h}\right\|_{0}^{2}+E_{I}\left(p^{h}, p^{h}-q^{h}\right)\right\}
$$

holds for every $q^{h} \in Q_{h}$.

Proof. Applying (4.5), we have

$$
\left(p^{h}-q^{h}, v^{h}\right)=a\left(u^{h}-u, v^{h}\right)+\left(p-q^{h}, v^{h}\right)+E_{I}\left(p^{h}, v^{h}\right) .
$$

Since the condition (4.7) is equivalent to the condition

we have

$$
\exists v^{h} \in V_{h}: v^{h}=q^{h} \text { and } \hat{x}_{h}\left\|v^{h}\right\|_{1} \leqq\left\|q^{h}\right\|_{0},
$$

$$
\left\|p^{h}-q^{h}\right\|_{0}^{2} \leqq M\left\|u-u^{h}\right\|_{1}\left\|p^{h}-q^{h}\right\|_{0} / \hat{\alpha}_{h}+\left\|p-q^{h}\right\|_{0}\left\|p^{h}-q^{h}\right\|_{0}+E_{I}\left(p^{h}, p^{h}-q^{h}\right) .
$$

Applying Young's inequality yields

$$
\left\|p^{h}-q^{h}\right\|_{0}^{2} \leqq C\left\{\left(\left\|u-u^{h}\right\|_{1} / \hat{Q}_{h}\right)^{2}+\left\|p-q^{h}\right\|_{0}^{2}+E_{I}\left(p^{h}, p^{h}-q^{h}\right)\right\} .
$$

The result (4.8) then follows from the triangular inequality.

The above result gives the estimate of $p-p^{h}$ in $L^{2}(\Omega)$-norm, and the rate of convergence is dominated by $1 / \hat{\alpha}_{h}$ if $\hat{\alpha}_{h}$ depends upon $h$, the mesh size. However, if the estimate of $p-p^{h}$ is considered in the dual space of $H_{0}^{1}(\Omega)$, then the following result can be obtained:

Theorem 4.3. Suppose that

(i) $\exists v_{I}^{h} \in V_{h} \quad$ s.t. $\left\|v-v_{I}^{h}\right\|_{0} \leqq M_{1} h\|v\|_{1}$,

and $\left\|v_{I}^{h}\right\|_{1} \leqq \hat{M}_{1}\|v\|_{1}$, for every $v \in H^{1}(\Omega)$, with

$$
\text { (ii) } \sup _{v \in H^{1}(\Omega)} \frac{E_{I}\left(p^{h}, v_{I}^{h}\right)}{\|v\|_{1}} \leqq M_{3} h^{\mu}, \quad \mu>0
$$

for every $p^{h} \in Q_{h}$.

Then, we have

$$
\left\|p-p^{h}\right\|_{1}^{*} \leqq M_{1} h\left\|p-p^{h}\right\|_{0}+M_{2}\left\|u-u^{h}\right\|_{1}+M_{3} h^{\mu}
$$

Proof. It follows from (4.5) that

$$
\begin{aligned}
\left(p-p^{h}, v\right) & =\left(p-p^{h}, v-v^{h}\right)+a\left(u-u^{h}, v^{h}\right)-E_{I}\left(p^{h}, v^{h}\right) \\
& \leqq\left\|p-p^{h}\right\|_{0}\left\|v-v^{h}\right\|_{0}+\hat{M}_{2}\left\|u-u^{h}\right\|_{1}\left(\left\|v-v^{h}\right\|_{1}+\|v\|_{1}\right)-E_{I}\left(p^{h}, v^{h}\right)
\end{aligned}
$$

Then applying (4.9) and (4.10) we have

$$
\left\|p-p^{h}\right\|_{1}^{*}=\sup _{v \in V} \frac{\left(p-p^{h}, v\right)}{\|v\|_{1}} \leqq M_{1} h\left\|p-p^{h}\right\|_{0}+M_{2}\left\|u-u^{h}\right\|_{1}+M_{3} h^{\mu}
$$




\section{An Example of Error Estimates}

Final error estimates of the penalty-finite element approximation for a specific choice of finite elements and schemes of numerical integration $I$ follow from the results of interpolation properties of finite element methods defined on restricted sets $K_{h}$ and $N_{h}$ instead of $V_{h}$ and $Q_{h}$, respectively. Thus, the results due to [9] must be recalled here. Let $U_{h}$ be a finite element approximation of a subspace $U$ of a Sobolev space $H^{m}(\Omega), m>0$, such that $H_{0}^{m}(\Omega) \subset U \subset H^{m}(\Omega)$. Let $\sum$ be the set of all nodal points of the finite element model which construct finite elements. Let $k$ be the order of the complete polynomial contained in each interpolation function associated at a nodal point of the model. Suppose that $g \in H^{m}(\Omega) \cap C(\bar{\Omega})$, and $\Omega$ is the domain for both approximation and the continuous problem. Then

Theorem 5.1. Let $v^{h}$ be the unique element in $U_{h}$ such that $v^{h}=v$ on $\sum$. Then if $v \in M, v^{h} \in M_{h}$ and

$$
\begin{gathered}
\left\|v-v^{h}\right\|_{r} \leqq C h^{\mu}\|v\|_{s} \\
\mu=\operatorname{Min}\{k+1-r, s-r\}
\end{gathered}
$$

where $C$ is a constant independent of $v$ and $h$,

$$
\begin{aligned}
M & =\left\{v \in H^{m}(\Omega): v-g \leqq 0 \text { a.e. in } \Omega\right\} \\
M_{h} & =\left\{v^{h} \in V_{h}: v^{h}\left(\sum\right)-g\left(\sum\right) \leqq 0\right\}
\end{aligned}
$$

and $r \leqq m<s$.

As an example, we will discuss error estimates of the approximation by three-node linear triangular elements and "trapezoid" rule of numerical integration described in Example 2, in Section 3. Let the domain $\Omega$ be divided uniformly by three-node triangular elements, and let the integration rule $I$ be defined as in Example 2. Let

$$
u \in H^{2}(\Omega), \quad g \in H^{2}(\Omega) \cap C(\bar{\Omega}), \quad p \in L^{2}(\Omega)
$$

From Theorem 3.3,

$$
\begin{aligned}
& \left\|u_{\varepsilon}^{h}-u^{h}\right\|_{1} \leqq C_{1} \varepsilon h^{-1} \\
& \left\|p_{\varepsilon}^{h}-p^{h}\right\|_{0} \leqq C_{2} \varepsilon h^{-2}
\end{aligned}
$$

From Theorem 4.1 and 5.1, we have

since

$$
\left\|u-u^{h}\right\|_{1} \leqq C_{3} h
$$

$$
\left|E_{I}\left(q^{h}, v^{h}\right)\right| \leqq \hat{C}_{3} h^{2}\left\|q^{h}\right\|_{0}\left\|v^{h}\right\|_{2} .
$$

From Theorem 4.2 and 5.1, 
applying

$$
\left\|p-p^{h}\right\|_{0} \leqq \hat{C}_{4}
$$

$$
\left|E_{I}\left(q^{h}, \hat{q}^{h}\right)\right| \leqq \hat{C}_{4}\left\|q^{h}\right\|_{0}\left\|\hat{q}^{h}\right\|_{0} .
$$

Condition (4.9) follows from the theory of interpolation of finite element methods. Moreover, it can be easily verified that

$$
\left|E_{l}\left(p^{h}, v_{I}^{h}\right)\right| \leqq C h\left\|p^{h}\right\|_{0}\left\|v^{h}\right\|_{1}
$$

Then condition (4.10) is satisfied with $\mu=1$. From Theorem 4.3,

i.e.

$$
\left\|p-p^{h}\right\|_{1}^{*} \leqq M_{1} \hat{C}_{4} h+M_{2} C_{3} h+C h
$$

$$
\left\|p-p^{h}\right\|_{1}^{*} \leqq C_{5} h
$$

It may extend similar arguments to $\left\|p_{\varepsilon}^{h}-p^{h}\right\|_{0}$ and then

$$
\left\|p^{h}-p^{h}\right\|_{1}^{*} \leqq \hat{C}_{2} \varepsilon h^{-1}
$$

Therefore, if $\varepsilon=h^{2}$,

$$
\left\|u-u_{\varepsilon}^{h}\right\|_{1}+\left\|p-p_{\varepsilon}^{h}\right\|_{1}^{*} \leqq C_{6} h
$$

Acknowledgements. The author is very grateful to Professor J. Tinsley Oden who encouraged the continuation of the present study. The author's thanks are also extended to Dr. Y.J. Song who participated in discussions on various points of the analysis. The author was supported by AFOSR grant F-49620-78-C-0083 during this work.

\section{References}

1. Aubin, J.P.: Estimate of the error in the approximation of optimization problems with constraints by problems without constraints. In: Control theory and the calculus of variation, (A.V. Balakrishnan, ed.), pp. 153-173 New York: Academic Press, 1979

2. Babuška, 1.: The finite element method with lagrange multipliers. Numer. Math. 20, 179-192 (1973)

3. Bercovier, M.: Perturbation of mixed variational problems. Application to mixed finite element method. RAIRO, Numerical Analysis 12, 211-236 (1978)

4. Brezis, H., Stampacchia, G.: Sur la regularite de la solution d'inequations elliptiques. Bull. Soc. Math. France 96: 153-180 (1968)

5. Brezzi, $F$ : On the existence, uniqueness and approximations of saddle-point problems arising from lagrangian multipliers. RAIRO, R. 2, 129-151 (1974)

6. Brezzi, F., Hager, W.W., Raviart, P.A.: Error estimates for the finite element solution of variational inequalities - Part 1: Primal theory. Numer. Math. 28, 431-443 (1977)

7. Brezzi, F., Hager, W.W., Raviart, P.A.: Error estimates for the finite element solution of variational inequalities - Part II: Mixed methods. Numer. Math. 31, 1-16(1978)

8. Glowinski, R., Lions, J.L., Trémolièces, R.: Analyse numérique des inéquations variationelles. Paris: Dunod, 1976

9. Falk, R.S.: Error estimates for the approximation of a class of variational inequalities. Math. Comput. 28, 963-971 (1974)

10. Falk, R.S.: Approximation of an elliptic boundary value problem with unilateral constraints. Rev. Française Automat. Informat. Recherche Opérationnelle R 2, 5-12 (1975) 
11. Kikuchi, N.: Convergence of a penalty method for variational inequalities. TICOM Report 79-16, The University of Texas at Austin, 1979

12. Lions, J.-L.: Quelques methodes de résolution des problèmes aux limites non linéaires. Paris: Dunod, 1969

13. Lions, J.-L.: Sur quelques questions d'analyse, de mécanique et de contrôle optimal. Montreal: Les Presses de l'Universite, 1976

14. Mosco, U., Strang, G.: One-sided, approximation and variational inequalities. Bull. Amer. Math. Soc., 80, 308-312 (1974)

15. Oden, J.T., Kikuchi, N., Song, Y.J.: An analysis of exterior penalty methods and reduced integration for finite element approximations of contact problems in incompressible elasticity. TICOM Report 80-2, The University of Texas at Austin, 1980

16. Temam, R.: Navier-Stokes equations. Amsterdam: North-Holland. 1977

Received October 17, 1979/June 18, 1980/September 16,1980 